Proceedings of the 45th International School and Conference on the Physics of Semiconductors "Jaszowiec" 2016, Szczyrk

\title{
Deterioration of Mechanical Properties of MBE-Grown, Metastable Semiconductor Layer with Time: the Case of Zinc Blende MnTe
}

\author{
S. Adamiak ${ }^{a, *}$, E. Dynowska ${ }^{b}$, A. Dziedzic $^{a}$, K. Szmuc ${ }^{a}$, E. Janik $^{b, \dagger}$, M. Wiater $^{b}$, \\ T. WOJTOWICZ ${ }^{b}$ AND W. SzUszKIEWICZ ${ }^{a, b}$ \\ ${ }^{a}$ Faculty of Mathematics and Natural Sciences, University of Rzeszów, S. Pigonia 1, PL-35310 Rzeszów, Poland \\ ${ }^{b}$ Institute of Physics, Polish Academy of Sciences, Aleja Lotnikow 32/46, PL-02668 Warsaw, Poland
}

Several few $\mu \mathrm{m}$ thick (001)-oriented, metastable MnTe layers with the zinc blende structure grown onto (001) GaAs substrate by MBE during different periods for the last twenty years were investigated by the scanning electron microscopy, atomic force microscopy, X-ray diffraction, and nanoindentation methods. A partial decomposition of the oldest investigated layers was demonstrated. An important decrease of Young's modulus from about $34 \mathrm{GPa}$ to about $17 \mathrm{GPa}$, resulting from a deterioration of the crystal structure of such layers, was found.

DOI: 10.12693/APhysPolA.130.1248

PACS/topics: 61.05.cp, 62.20.--x, 64.60.My

\section{Introduction}

An enormous development of the non-equilibrium growth techniques in solid state physics observed for many years results from both a presence of numerous new physical effects, related to low-dimensional character of investigated objects and from several needs of contemporary solid state industry. Among attracting a lot of attention, low-cost methods serving for a thin layer deposition such techniques as, e.g., vacuum evaporation electrodeposition, spray pyrolysis, etc. should be mentioned. Much more sophisticated and the same time much more expensive methods like, e.g., the molecular beam epitaxy (MBE) or the atomic layer deposition (ALD) are applied for the growth of low-dimensional structures, required for selected devices or applications. From one side there exists an interest in the growth and characterization of low-dimensional semiconductor object in the same crystal phase, as that of its bulk equivalent. On the other side, an application of technique like MBE makes it possible to create thin layers or quantum structures of materials with quite a new crystal phase, which does not exist in nature in the bulk form or cannot be obtained by the equilibrium growth methods. The materials obtained in such a manner often exhibit new, required physical properties, corresponding to particular needs of given device or application. The success of non-equilibrium growth method is related to well selected crystal symmetry and lattice parameter values of both a substrate and possible buffer layer, applied for a given problem in technology. The proper choice of substrate and buffer materials due

*corresponding author; e-mail: sadamiak@ur.edu.pl

${ }^{\dagger}$ Present address: Institute of Experimental Physics, Faculty of Physics, University of Warsaw, L. Pasteura 5, PL-02093 Warsaw, Poland to a presence of a compressive or tensile strain introduced in the grown layer stabilize its structure. For some particular purposes like, e.g., the space applications related to the space missions etc. the long lifetime of electronic elements and other devices is a crucial problem. Up to our knowledge up to now the stability of "metastable" form of semiconductor compounds were out of interest and the relevant literature does not exist at all. Under the circumstances this problem has been selected as a topic of present studies.

One from the best known semiconducting compounds widely investigated in both its stable and metastable crystal phase is magnetic semiconductor MnTe. The MnTe phase diagram can be found in [1]. The stable MnTe crystal phase exhibits a hexagonal structure of NiAs type ([2,3] and references therein). The metastable MnTe phase crystallizes in the zinc blende structure, this form of crystal has been obtained for the first time in [4]. Due to its particular antiferromagnetic order of AFIII type the zinc blende MnTe was intensively studied since 1989 first in the form of thin layers (see, e.g., [4-7]) and next as a constituent of superlattices and other low-dimensional quantum structures. In particular, three elastic contants and bulk modulus values of zinc blende MnTe were obtained by the Brillouin light scattering, the Raman scattering, and ab initio calculations ([8] and references therein). Under the circumstances the zinc blende MnTe seemed to be a perfect model object for planned studies.

\section{Experimental details}

The several few micrometer thick, zinc blende, (001)oriented MnTe layers were grown by MBE technique on (001)GaAs substrate covered with a thin CdTe buffer in the period 1996-2016. All of them were obtained with the use of the EPI 620 Molecular Beam Epitaxy dedicated for II-VI compounds growth. The perfect structure quality 
of all layers after the growth was confirmed by X-ray diffraction (XRD) using a standard, medium-resolution laboratory diffractometer and $\mathrm{Cu} K_{\alpha_{1}}$ radiation with the wavelength $\lambda=1.54046 \AA$. The structure quality was checked for the second time very recently by XRD using the X'Pert MPD Philips (PANalytical) diffractometer equipped with the $\mathrm{Cu}$ X-ray tube and (111)-oriented Ge Johansson monochromator (the same $\mathrm{Cu} K_{\alpha_{1}}$ radiation was applied).

The surface topography of MnTe layers was investigated by the atomic force microscopy (AFM) technique. The room temperature AFM imaging in contact mode was performed in air using the CSM Instrument microscope. For all sample their surfaces were also investigated with the use of scanning electron microscopy (SEM). Finally, the Young modulus was determined for investigated layers by nanoindentation measurements. The parameters of applied load were as follows: maximum load $1 \mathrm{mN}$, linear change of the load during application or removal of the load $0.033 \mathrm{mN} / \mathrm{s}$, application time of the maximum load $30 \mathrm{~s}$.

\section{Results and discussion}

The X-ray diffraction pattern taken for $6.5 \mu \mathrm{m}$ thick MnTe layer, grown in 2016 shown in Fig. 1 confirmed its perfect zinc blende structure quality. The angular positions of MnTe-related Bragg peaks correspond to the value of zinc blende lattice parameter $a=0.6338 \mathrm{~nm}$, determined for this crystal phase long time ago [6]. The analogous diffraction patterns, taken for 20 years old samples (not shown) demonstrated a presence of several peaks with much smaller intensity, identified as those corresponding to various hexagonal Te or manganese oxides Bragg reflections. Such findings evidenced a partial decomposition of investigated layers with time due to a metastable character of crystal phase selected for the growth and suggested possible deterioration of mechanical properties of these layers. The results of measurements by the AFM demonstrated a partial modification of the layer surface and an important increase of the surface roughness (Fig. 2). The results of energy dispersive X-ray (EDX) measurements (not shown) indicated the presence of a residual $\mathrm{C}$ content in all investigated layers. It is not a surprising effect. A careful analysis of results of EDX or secondary ion mass spectroscopy (SIMS) measurements always detects a presence of a small $\mathrm{C}$ quantity in thin layer or low-dimensional structure due to some sample surface contamination by carbon during the growth or due to the sample contact with air. However, a noticeably higher $\mathrm{C}$ content was found for the modified surface of the elder sample, than that for the perfect surface of recently grown MnTe layer. This finding suggests that in the former case surface is more developed (e.g., as a result of partial decomposition of the top surface layer into separate slices) than in the latter one. From the results of nanoindentation measurements the Young modulus was determined for all layers. The typical nanoindentation depth under the

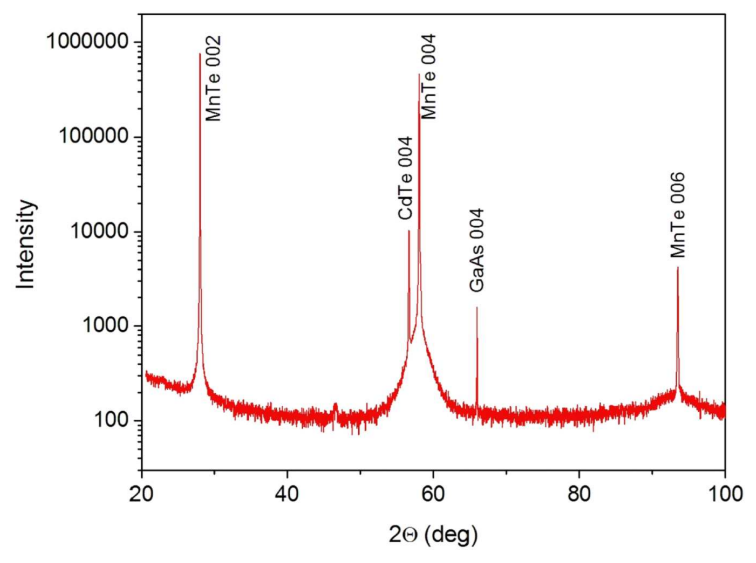

Fig. 1. Typical diffraction pattern taken for zinc blende MnTe layer after the MBE growth.
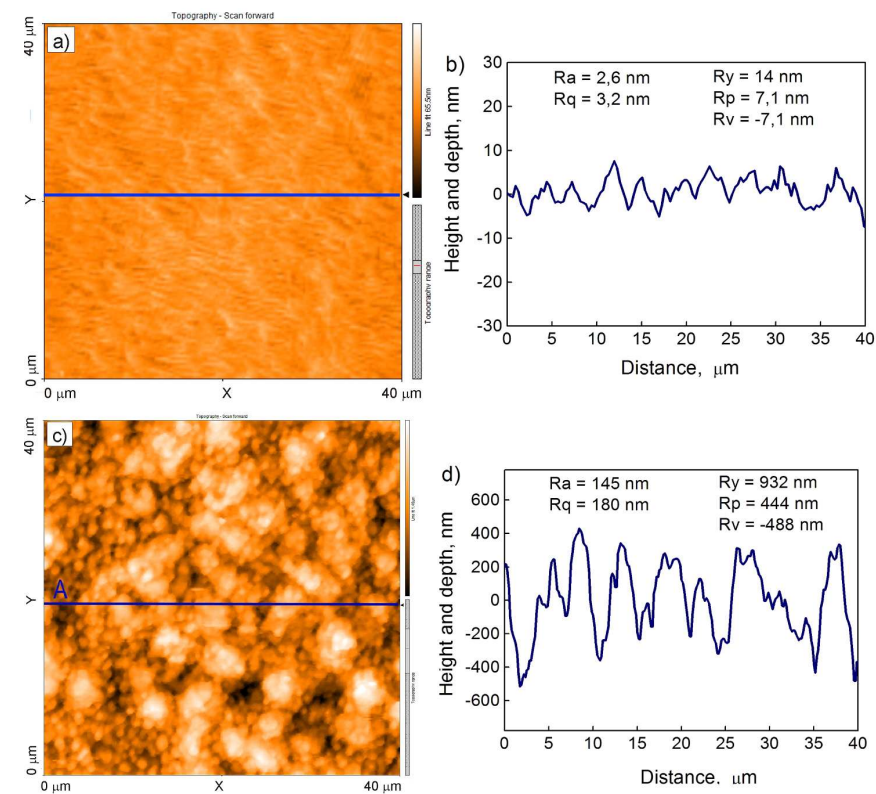

Fig. 2. Surface topography of MBE-grown, zinc blende (001)-oriented MnTe layer determined by AFM method. (a) Top view of $6.5 \mu \mathrm{m}$ thick perfect layer just after the growth. (b) Surface profile for the same layer along the line shown in (a). (c) Top view of degraded (20 year old) $5 \mu \mathrm{m}$ thick layer. (d) Surface profile for this degraded layer along the line shown in (c).

experimental conditions applied in these measurements was close to $250 \mathrm{~nm}$. In order to measure the mechanical properties related to the layer only a commonly used rule is to limit the indentation depth to less than $10 \%$ of the layer thickness [9]. The thickness of investigated layers in all cases was at least 20 times higher, than this depth, so possible influence of the mechanical properties of the substrate on the Young modulus of various MnTe layers should be excluded. The value of the Young modulus determined for a perfect layer $E=34 \pm 2 \mathrm{GPa}$ was slightly smaller than that corresponding to $\mathrm{CdTe}$, reported in the literature $[10,11]$. On the contrary, the Young modulus for 20 year old layers was significantly lower and equal to 
$E=17 \pm 2$ GPa only. It should be stressed that 10 year old MnTe layer did not exhibit yet a partial decomposition or a deterioration of its mechanical properties with time which suggests relatively long period of a stability of metastable phase forced by a strain applied during the MBE growth.

\section{Conclusion}

The deterioration of structure and mechanical properties of MBE-grown MnTe layer in its metastable phase started several years (at least 10) after its growth. The Young modulus value $E=34 \pm 2 \mathrm{GPa}$ was determined for the perfect zinc blende MnTe layer for the first time. This value for 20 year old MnTe layer is much smaller and equals $E=17 \pm 2 \mathrm{GPa}$.

\section{Acknowledgments}

This work was supported in part by National Science Centre (Poland) through grant UMO2014/13/B/ST3/04393.

\section{References}

[1] M.E. Schlesinger, J. Phase Equil. 19, 591 (1998).

[2] W. Szuszkiewicz, E. Dynowska, B. Witkowska, B. Hennion, Phys. Rev. B 73, 104403 (2006).
[3] R. Minikayev, E. Dynowska, B. Witkowska, A.M.T. Bell, W. Szuszkiewicz, X-Ray Spectrom. 44, 394 (2015).

[4] S.M. Durbin, J. Han, O. Sungki, M. Kobayashi, D.R. Menke, R.L. Gunshor, Q. Fu, N. Pelekanos, A.V. Nurmikko, D. Li, J. Gonsalves, N. Otsuka, Appl. Phys. Lett. 55, 2087 (1989).

[5] T.M. Giebultowicz, P. Klosowski, N. Samarth, H. Luo, J.K. Furdyna, J.J. Rhyne, Phys. Rev. B 48, 12817 (1993).

[6] E. Janik, E. Dynowska, J. Bak-Misiuk, M. Leszczynski, W. Szuszkiewicz, T. Wojtowicz, G. Karczewski, A.K. Zakrzewski, J. Kossut, Thin Solid Films 267, 74 (1995).

[7] B. Hennion, W. Szuszkiewicz, E. Dynowska, E. Janik, T. Wojtowicz, Phys. Rev. B 66, 224426 (2002).

[8] P. Djemia, Y. Roussigne, A. Stashkevich, W. Szuszkiewicz, N. Gonzalez Szwacki, E. Dynowska, E. Janik, B.J. Kowalski, G. Karczewski, P. Boguslawski, M. Jouanne, J.F. Morhange, Acta Phys. Pol. A 106, 239 (2005).

[9] S. Simunkova, O. Blahova, I. Stepanek, J. Mater. Process. Technol. 133, 189 (2003).

[10] M. Pang, D.F. Bahr, K.G. Lynn, Appl. Phys. Lett. 82, 1200 (2003).

[11] G. Purcek, E. Bacaksiz, I. Miskioglu, J. Mater. Process. Technol. 198, 202 (2008). 